\title{
Stimulus accessibility and music theory/therapy
}

\author{
Anna Yu Wang \\ Graduate School of Arts and Sciences, Harvard University, Cambridge, MA, United States \\ Corresponding author: anna wang@g.harvard.edu \\ Published 16 December 2021; https://doi.org/10.18061/FDMC.2021.0025 \\ Author video presentation and/or other conference material: https://doi.org/10.17605/OSF.IO/GXF6R
}

\begin{abstract}
This paper draws on music therapeutic, neuroscientific, and philosophical literature to posit three aspects of musical engagement that qualify music as an unusually accessible stimulus: 1) audition as a means of self-orientation 2) music's instigation of self-referential thought, and 3) the lower threshold required for processing musical meaning compared to linguistic meaning. This accessibility renders music a promising therapeutic stimulus for people living with a disorder of consciousness or other cognitive disorders, as clinical studies suggest. Moreover, this paper argues that culturally sensitive music theory and cognition can help maximize music's therapeutic potential by clarifying the variables that influence the accessibility of musical stimuli. Specifically, by complicating the research findings from participant cohorts dominated by members of Western, Educated, Industrialized, Rich, and Democratic (WEIRD) societies, music theory and cognition can illuminate how cultural context impacts the manner and extent to which listeners derive therapeutic benefit from musical structures. This suggests that there is fertile ground for future collaborative work between music therapists, cognitivists, and theorists.
\end{abstract}

KEYWORDS: music therapy, disorders of consciousness, accessibility, music theory, world music

\section{Introduction}

Disorders of consciousness (DOC) is an umbrella term that encompasses comatose, vegetative, and minimally conscious states, each of which severely reduces a person's ability to engage with their surroundings cognitively and physically. At the mild end of the DOC spectrum a person may not be able to comprehend spoken commands, while those more gravely affected require life-sustaining therapy and exhibit only the scarcest signs of awareness (Giacino et al., 2013). While pharmaceutical treatments for DOC remain limited, a growing body of literature points optimistically to the rehabilitative potential of music therapy (Grimm and Kreutz, 2018; Magee et al., 2016; Sun and Chen, 2015). This paper frames music's therapeutic efficacy as a matter of stimulus accessibility, which has been defined by Hansen (1959) as "the potential for interaction."

Along these lines, Perrin et al. (2015) have argued that "stimulus selection is critical for an accurate evaluation of the state of a patient with a disorder of consciousness as it determines the level of processing that a patient can have with his/her environment" (1). Identifying stimuli that are accessible enough to elicit cognitive processing and an agential response from DOC patients can be of seminal importance for their recovery. Patient responsiveness may improve the likelihood of an accurate diagnosis - with misdiagnosis potentially leading to substantial differences in the care team's allocation of rehabilitative and even lifesustaining resources (Edlow et al., 2017) - and it may help strengthen the patient's remaining neural connections (Langille and Brown, 2018).

This paper posits three reasons that qualify music as an unusually accessible stimulus for DOC patients: 1) the relationship between audition and self-orientation 2) music's instigation of self-referential thought, and 3) the lower threshold required for processing musical meaning compared to linguistic meaning. Clarifying the mechanism by which music is accessible to those with limited cognitive function will help clinicians more strategically harness music's therapeutic applications for people living with DOC and potentially other neurocognitive disorders such as dementia (El Haj et al. 2012).

This paper also draws on recent research in music theory and cognition to posit that not all music is equally accessible to every patient, and that accessibility depends on the degree of synergy that the chosen musical stimulus strikes with the patient's listening habits - those habits being socially and culturally formed. This adds a therapeutic imperative to the accumulating list of reasons for which the cultural scope of music theoretical and cognitive research should be expanded beyond the perceptual norms and preferred repertoires of WEIRD listeners-listeners from Western, Educated, Industrialized, Rich, and Democratic societies (Jacoby et al., 2020; Henrich et al., 2010). 


\section{Music as an Accessible Stimulus}

From the standpoints of diagnosis and recovery, it is crucial that DOC patients are invited to respond with their highest level of cognitive facility. For this, the appropriate selection of stimuli is of critical importance. The following broadly theorizes three ways that music represents an unusually accessible stimulus for individuals with limited consciousness on the grounds that being self-oriented within one's surroundings and having self-referential thought are requisites of conscious activity (Damasio, 1999; Morin, 2006; Kotchoubey, 2018).

\section{Audition and Self-Orientation}

Kotchoubey et al. (2015) collated ample neuroscientific evidence which suggest that audition implicates not only hearing but self-orientation. For example, they note that most of the input into the auditory cortex does not come directly from acoustic sub-cortical regions but from other cortices in the brain. They further point out that unlike the visual cortex, which pairs complex stimuli down to its basic components, the auditory system works on both complex and simple stimuli in tandem at nearly all levels of processing. For example, neurons on the auditory belt are not responsible for simple frequencies, but for harmonic spectra (Rauschecker, 1997). Moreover, upon observing mismatch negativity (MMN) elicited in the auditory cortex, Näätänen et al. (2001) speculated that the auditory cortex is also a site of "primitive" cognitive activity whereby sound patterns are tracked and anticipated. The auditory cortex's topdown heavy reception of information, its preference (in some regions) for complex tones rather than pure sine tones, and its ability to parse sonic patterns imply that the auditory cortex is more than a sensory processor. Rather, it performs a more holistic and associative task, appearing to be a site of context construction and of selfpositioning within an acoustic environment.

In this sense, audition can be likened to a form of proprioception, a haptic feedback which results in the awareness of oneself in relation to one's surrounding context. Music, then, as an auditory stimulus, taps into the proprioceptive system by which one gains awareness of the surrounding environment. Along these lines, Acitores (2011) drew on James Gibson's, Mark Johnson's, and Gerald Edelman's theories that consciousness requires proprioceptive awareness of the external environment to argue that conscious awareness resides within the body itself (as opposed to only in the "mind" in a mind/body dualist framework). She then connected this embodied mode of interacting with the world to the inextricability of body schema from musical engagement, as elaborated in Cox's (2011) mimetic hypothesis. Taken altogether, audition, the body, and music are related in their ability to foreground one's physical relationality with the environment, which in turn is an indispensable ingredient within consciousness and responsiveness.

\section{Music and Self-Referentiality}

Just as proprioception is a necessary condition for awareness, self-referential thought is necessary for the formation of conscious, agential responses. As such, neuroscientists working on DOC have devoted significant labor to the study of the default mode network (DMN) (e.g. Mäki-Marttunen et al., 2016; He et al., 2015; Bodien et al., 2019). The DMN is a network of neural structures hypothesized to be directly implicated in self-referential thought (Davey et al., 2016; Ino et al., 2011). Most recently, Bodien et al. (2019) studied the relationship between an intact DMN and a phenomenon known as covert consciousness. They concluded that while the DMN is a necessary condition for consciousness, it is not a sufficient condition: they found that there were some people with intact DMNs who did not exhibit conscious behavior. Nevertheless, there is much evidence that self-relevant stimuli are more cognitively accessible (Kempny et al., 2018; Perrin et al., 2015). For example, Howarth and Ellis (1961) found that there is a lower activation threshold (less energy required) for the perception of one's own name versus a random other first name. Given the limited neural resources of a DOC patient, self-referential stimuli appear to yield a higher likelihood of patient engagement because they require less mental exertion.

Several studies indicate that music is one type of self-referential stimulus. El Haj et al. (2012) showed that people with mild onset of Alzheimer's Disease were able to recollect more details about a past memory during an autobiographical interview after being played Vivaldi's "Spring." They subsequently found that the effect is amplified when music of the participant's own preference is played. Verger et al. (2014) also showed that DOC patients performed better on the Coma Recovery Scale-Revised (CRS-R) - the "gold standard" for determining consciousness in DOC patients - after listening to music, suggesting that music appreciably facilitates a patient's cognitive functioning. This phenomenon might be partially accounted for by the 
nature of musical semiosis - that is, musical meaning making - as described in the following section.

\section{The Semiosis of Music Versus Language}

Music, like language, is a semiotic system - a system in which meanings are extracted from signs. The process of translating signs into meanings is mediated by an interpreting mind and can result in traces of the interpreter's inner constructs - including their memories, predispositions, and cultural backgroundbeing encoded into the extracted meaning. Just as mirrors serve to reflect our outer appearances, semiotic systems can serve as reflective surfaces of our inner constructs.

However, the degree of "reflectivity" elicited by a semiotic system is not the same across all stimuli. Rather, it depends on the nature of the sign being parsed. Music, for instance, is composed primarily of Peircean "indices" (Atkin, 2013) - signs that do not come with a codified lexicon, and whose meanings are created on a case-by-case basis in relation to the interpreter's experiences (Zbikowski, 2011). Music is therefore a rather reflective stimulus. Meanwhile, language is not as reflective because it consists mainly of Peircean "symbols"-signs whose meanings are already predefined by convention (Table 1). In other words, while the semiosis of language requires engagement with an externalized database of meanings - found in a dictionary-musical semiosis draws heavily on an internalized database for its meanings, as furnished by one's own memories and experiences. Therefore, musical stimuli theoretically constitute the more direct and unfettered pathway to the mind.

The implications of this hypothesis, if it is empirically supported, are substantial. For one, there would be reason to redesign the CRS-R, which currently relies heavily on the patient's ability to comprehend verbal commands. We might instead move towards, or at least normatively incorporate, diagnostic tests that include musical stimuli such as the MATADOC (Magee et al., 2014), which appear to elicit higher degrees of arousal from DOC patients than CRS-R tasks (Bodine et al., 2020). Moreover, support for this hypothesis would also establish a concrete direction for the development of future DOC treatments: treatments would, as much as possible, capture and project aspects of the pre-injured mind back to the patient.
Table 1: Descriptions of a Peircean symbol and index.

\begin{tabular}{|l|l|}
\hline $\begin{array}{l}\text { Peircean Symbol } \\
\text { (Many linguistic signs) }\end{array}$ & $\begin{array}{l}\text { Peircean Index } \\
\text { (Many musical signs) }\end{array}$ \\
\hline Arbitrary, Conventional & Logical relationship \\
\hline $\begin{array}{l}\text { Defined through social } \\
\text { agreement }\end{array}$ & $\begin{array}{l}\text { Grounded in personal } \\
\text { experience }\end{array}$ \\
\hline
\end{tabular}

\section{Possibilities for Collaboration between Music Therapists, Cognitivists and Theorists}

Music therapy training generally requires practitioners to be fluent in Western musical idioms and Western harmonic instruments like piano and guitar (Wigram et al. 2002; Howland 2017). Practitioners are not typically required to develop a refined understanding of how music is structured and expressed in other cultures. This might be attributed, at least in part, to the academic standard set by music therapy's older sibling fields of music theory and cognition, which have also historically centered around the principles and perception of Western music.

However, if the efficacy of music therapy is rooted in music's accessibility as a stimulus, and accessibility is about the potential for interaction between the stimulus and the listener, then accessibility cannot be generalized across all listeners. Rather, to achieve optimal accessibility, musical stimuli and their strategic use must be tailored to the unique social and cultural bearings of each listener. While it is well within music therapy's modus operandi to find musical stimuli that are meaningful to individual patients, the current institutionalized knowledge about musics beyond the West is insufficient to truly allow for the strategic therapeutic use of such musics. For patients whose habits of listening differ from those represented in conventional, Western-focused music cognitive and theoretical research, the optimization of stimulus accessibility depends not only upon the choice of repertoire, but also on a nuanced understanding of how the patient is attending to the musical structures found in that repertoire.

Recently, a small but growing corpus of music theoretical and cognitive research has begun to challenge the assumed pan-applicability of Western listening habits by showing that the same musical structure can trigger very different listener responses depending on cultural context. For example, Margulis et al. (2019) showed that while one musical example prompted images of play for audiences in Dimen, China, the same example conjured frightening images for 
audiences in Arkansas, USA. Working along the parameters of pitch and rhythmic perception, McDermott et al. (2016) demonstrated that the degree of pleasure that isolated dissonant sounds invoke for a listener varies across cultures, while Jacoby and McDermott (2017) found that rhythmic priors may also be largely culturally determined. In the realm of music theory, scholars have attempted to trace divergent listening habits back to specific cultural and ideological forces (Yu Wang, in press; Stover, 2020; Goldberg, 2020; Tse and Wong, 2020).

This type of research is uncovering radical differences in the way musical sound is conceptualized and attended to, suggesting that a musical stimulus should not be expected to invoke similar experiences nor to present the same degree of accessibility to all listeners. In order to maximize the accessibility of musical stimuli, it is necessary that we answer the following questions: What are the factors that render a particular musical structure more or less accessible (i.e. along the parameters of self-orientation, selfreferentiality, and memory invocation) to a given listener? How can we strategically select culturally resonant musical stimuli to elicit a desired listener response?

The onus is largely on music theorists and cognitivists, as scholars with a critical ear towards the perceptual implications of musical structure, to be sensitive to the diversity of ways in which musical structures can be attended to. We need culturally sensitive contributions to music theory and cognition not only for the lasting rigor of these fields, but for the benefit of patients and their loved ones who are relying on the restorative effects of music therapy. This line of reasoning encourages music cognitivists and theorists to think of music therapists as both a potential audience for our research, and also perhaps as future collaborators.

\section{Conclusion}

Accessibility of stimuli is particularly critical for patients with DOC, for whom the primary medical challenge is to restore their ability to interact with their surroundings. Music cognitivists and theorists are poised to augment the efficacy of music therapy by contributing research on the factors by which a musical stimulus is made more or less accessible to a given listener, as impacted by cultural context. When these conditions are known, music therapists will be better equipped to design tailored therapy plans for patients with DOC and potentially a broader array of cognitive disorders. There are thus consequential grounds for cooperation between music theorists, cognitivists, and therapists.

\section{Acknowledgements}

I am grateful to Brian Harris, Alexander Rehding, and Leo Hanyi $\mathrm{Yu}$ for their constructive comments on this paper.

\section{References}

Acitores, A.P. (2012). In Clarke, D. \& Clarke E. (Eds.), Music and consciousness (pp. 215-230). Oxford University Press.

Atkin, A. 2013. Peirce's Theory of Signs. In E.N. Zalta (ed.), The Stanford encyclopedia of philosophy. Metaphysics Research Lab, Stanford University. https://plato.stanford.edu/archives/sum2013/entries/peirc e-semiotics/.

Bodien, Y.G., Threlkeld, Z.D., \& Edlow, B.L. (2019). Default mode network dynamics in covert consciousness. Cortex, 119 571-574.

https://doi.org/10.1016/j.cortex.2019.01.014

Bodine, C., Seu, A., Roth, E.A., Wegener, E.E., \& Magee, W.L. (2020). Examining the functionality of the MATADOC with the CRS-R: A pilot study. The Journal of Music Therapy, 57(4), 432-454. https://doi.org/10.1093/jmt/thaa010

Cox, A. (2011). "Embodying Music: Principles of the Mimetic Hypothesis.” Music Theory Online, 17(2): np. https://doi.org/10.30535/mto.17.2.1

Damasio, A. (1999). The feeling of what happens: Body and emotion in the making of consciousness. New York: Harcourt Brace.

Davey, C.G., Pujol, J., \& Harrison, B.J. (2016). Mapping the self in the brain's default mode network. NeuroImage, 132 , 390-397. https://doi.org/10.1016/i.neuroimage.2016.02.022

Edlow, B.L., Camille, C., Spencer, C.A., Chu, C.J., Bodien, Y.G., O'Connor, K. L., Hirschberg, R.E., Hochberg, L.R., Giacino, J.T., Rosenthal, E.S., \& Wu, O. (2017). Early detection of consciousness in patients with acute severe traumatic brain injury. Brain, (140)9, 2399-2414. https://doi.org/10.1093/brain/awx176

El Haj, M., Postal, V., \& Allain, P. (2012). Music enhances autobiographical memory in mild Alzheimer's Disease. Educational Gerontology, 38(1), 30-41. https://doi.org/10.1080/03601277.2010.515897

Giacino, J.T., Katz, D.I., \& Whyte, J. (2013). Neurorehabilitation in disorders of consciousness. Seminars in Neurology, 33(2), 142-156. https://doi.org/10.1055/s-0033-1348960

Grimm, T., \& Kreutz, G. (2018). Music interventions in disorders of consciousness (DOC) - A systematic review. Brain Injury, 32(6), 704-714. 


\section{https://doi.org/10.1080/02699052.2018.1451657}

Goldberg, D. (2020). What's the meter of Elenino Horo? Rhythm and timing in drumming for a Bulgarian folk dance. Analytical Approaches to World Music, 7(2), 69107.

Hansen, W.G. (1959). How accessibility shapes land use. Journal of the American Institute of Planners, 25(2), 7376. https://doi.org/10.1080/01944365908978307

He, J.H., Cui, Y., Song, M., Yang, Y., Dang, Y.Y., Jiang, T.Z, \& Xu, R.X. (2015). Decreased functional connectivity between the mediodorsal thalamus and default mode network in patients with disorders of consciousness. Acta Neurologica Scandinavica, 131(3), 145-151. https://doi.org/10.1111/ane.12299

Henrich, J., Heine, S. J., \& Norenzayan, A. (2010). The weirdest people in the world? Behavioral and Brain Sciences, 33, 61-135. https://doi.org/10.1017/S0140525X0999152X

Howarth, C.I., Ellis, K. (1961). The relative intelligibility threshold for one's own name compared with other names. Q. J. Exp. Psychol., 13 (4), 236-239. https://doi.org/10.1080/17470216108416500

Howland, K.M. (2016). Music therapy. In Encyclopedia Britannica. https://www.britannica.com/topic/musictherapy.

Ino, T., Nakai, R., Azuma, T., Kimura, T., \& Fukuyama, H.. (2011). Brain activation during autobiographical memory retrieval with special reference to default mode network. The Open Neuroimaging Journal, 5, 14-23. https://doi.org/10.2174/1874440001105010014

Jacoby, N., Margulis, E.H., Clayton, M., Hannon, E., Honing, H., Iverson, J., Klein, T.R., Mehr, S.A., Pearson, L., Peretz, I., Perlman, M., Polak, R., Ravignani, A., Savage, P.E., Steingo, G., Stevens, C.J., Trainor, L., Trehub, S., Veal, M. \& Wald-Fuhrmann, M. (2020). Cross-Cultural work in music cognition: Challenges, insights and recommendations. Music Perception, 37(3), 185-195. https://doi.org/10.1525/mp.2020.37.3.185

Jacoby, N., McDermott, J.H. (2017) Integer ratio priors on musical rhythm revealed cross-culturally by iterated reproduction. Current Biology, 27, 359-370. https://doi.org/10.1016/j.cub.2016.12.031

Kempny, A.M., James, L., Yelden, K., Duport, S., Farmer, S.F., Playford, E.D., \& Leff, A.P. (2018). Patients with a severe prolonged Disorder of Consciousness can show classical EEG responses to their own name compared with others' names. NeuroImage, 19, 311-319. https://doi.org/10.1016/j.nicl.2018.04.027

Kotchoubey, B. (2018). Human Consciousness: Where Is It From and What Is It for. Frontiers in Psychology, 9, 567. https://doi.org/10.3389/fpsyg.2018.00567

Kotchoubey, B., Pavlov, Y.G., \& Kleber, B. (2015). Music in Research and Rehabilitation of Disorders of
Consciousness: Psychological and Neurophysiological Foundations. Frontiers in Psychology, 6, 1763. https://doi.org/10.3389/fpsyg.2015.01763

Langille, J.J., \& Brown, R.E. (2018). The synaptic theory of memory: A historical survey and reconciliation of recent opposition. Frontiers in Systems Neuroscience, 12, 1-15. https://doi.org/10.3389/fnsys.2018.00052

Magee, W.L., \& O'Kelly, J. (2015). Music therapy with disorders of consciousness: Current evidence and emergent evidence-based practice. Annals of the New York Academy of Sciences, 13371(1), 256-62. https://doi.org/10.1111/nyas.12633

Magee, W.L., Siegert, R.J., Daveson, B.A., Lenton-Smith, G., \& Taylor, S.M. (2014). Music therapy assessment tool for awareness in disorders of consciousness (MATADOC): Standardisation of the principle subscale to assess awareness in patients with disorders of consciousness. Neuropsychological Rehabilitation, 24(1), 101-124. https://doi.org/10.1080/09602011.2013.844174

Mäki-Marttunen, V., Castro, M., Olmos, L., Leiguarda, R., \& Villarreal, M. (2016). Modulation of the default-mode network and the attentional network by self-referential processes in patients with disorder of consciousness. Neuropsychologia, $\quad 82, \quad 149-160$. https://doi.org/10.1016/j.neuropsychologia.2016.01.022

Margulis, E.H., Wong, P.C.M., Simchy-Gross, R. \& McAuley, J.D. (2019). What the Music Said: Narrative Listening Across Cultures. Palgrave Communications, 5, 146. https://doi.org/10.1057/s41599-019-0363-1

McDermott, J.H., Schultz, A.F., Unduragga, E.A. \& Godoy, R.A. (2016) Indifference to dissonance in native Amazonians reveals cultural variation in music perception. Nature, 535, 547-550. https://doi.org/10.1038/nature18635

Morin, A. (2006). Levels of consciousness and selfawareness: A comparison and integration of various neurocognitive views. Consciousness and Cognition, $15(2)$, 358-371. https://doi.org/10.1016/j.concog.2005.09.006

Näätänen, R., Tervaniemi, M., Sussman, E., Paavilainen, P. \& Winkler, I. (2001). 'Primitive intelligence' in the auditory cortex. Trends in Neurosciences, 24(5), 283288. https://doi.org/10.1016/S0166-2236(00)01790-2

Perrin, F., Castro, M., Tillmann, B. \& Luauté, J. "Promoting the use of personally relevant stimuli for investigating patients with disorders of consciousness. Frontiers in Psychology 6 (2015). https://doi.org/10.3389/fpsyg.2015.01102

Rauschecker, J.P., Tian, B., Pons, T. \& Mishkin, M. (1997). Serial and parallel processing in rhesus monkey auditory cortex. J. Comp. Neurol. 382, 89-103. https://doi.org/10.1002/(SICI)1096-9861(19970526)382 


\section{$: 1<89::$ AID-CNE6 $>3.0$. CO $; 2-\mathrm{G}$}

Sun, J. \& W. Chen. (2015). Music therapy for coma patients: Preliminary results. European Review for Medical and Pharmacological Sciences, 19(7), 1209-18.

Stover, C. (2020). Contextual theory, or theorizing between the discursive and the material. Analytical Approaches to World Music, 7(2), 13-40.

Tse, C.Y. and Wong, C.F. (2020). Metrical structure and freedom in qin music of the Chinese literati. Analytical Approaches to World Music, 8(2), 164-194.

Verger, J., Ruiz, S., Tillmann, B., Ben Romdhane, M., De Quelen, M., Castro, M., Tell, L., Luauté, J. \& Perrin, F. (2014). Effets bénéfiques de la musique préférée sur les capacités cognitives des patients en état de conscience minimale. Revue Neurologique, 170(11), 693-99. https://doi.org/10.1016/j.neurol.2014.06.005

Zbikowski, Lawrence M. (2011). Music, Language, and Kinds of Consciousness. In D. Clarke \& E. Clarke (Eds.), Music and consciousness (pp. 179-192). Oxford University Press. https://doi.org/10.1093/acprof:oso/ $\underline{9780199553792.003 .0058}$ 